ALEA, Lat. Am. J. Probab. Math. Stat. 14, 427-444 (2017)

DOI: 10.30757/ALEA.v14-23

\title{
The number of cycles in random permutations without long cycles is asymptotically Gaussian
}

\author{
Volker Betz and Helge Schäfer \\ Technische Universität Darmstadt \\ Fachbereich Mathematik \\ Schlossgartenstraße 7 \\ D-64289 Darmstadt, Germany. \\ E-mail address: \{ betz , hschaefer \} @mathematik.tu-darmstadt.de
}

\begin{abstract}
For uniform random permutations conditioned to have no long cycles, we prove that the total number of cycles satisfies a central limit theorem. Under additional assumptions on the asymptotic behavior of the set of allowed cycle lengths, we derive asymptotic expansions for the corresponding expected value and variance. In contrast to the case of uniform permutations, the asymptotic mean and variance are not logarithmic in the system size.
\end{abstract}

\section{Introduction}

It is a classical result about random permutations proved by Gončarov (1962) that the number of cycles of a uniform random permutation is asymptotically Gaussian after suitable normalization. More precisely, for a permutation $\sigma \in \mathcal{S}_{n}$, write $c_{j}(\sigma)$ for the number of cycles of length $j$ in $\sigma$ and $C(\sigma)=\sum_{j=1}^{\infty} c_{j}(\sigma)$ for the total number of cycles. Let $\mathbb{P}_{n}$ denote the uniform distribution on $\mathcal{S}_{n}$, then for all $y \in \mathbb{R}$,

$$
\lim _{n \rightarrow \infty} \mathbb{P}_{n}(C-\log n \leq y \sqrt{\log n})=\Phi(y)
$$

where $\Phi$ is the distribution function of the standard normal distribution.

A particularly instructive proof of this result was given in Shepp and Lloyd (1966), where it is observed that the joint distribution of the $c_{j}, j \leq n$, under $\mathbb{P}_{n}$ is equal to the distribution of independent random variables $\tilde{c}_{j}, j<\infty$, conditional on $\sum j \tilde{c}_{j}=n$, where $\tilde{c}_{j}$ is Poisson distributed with parameter $z^{j} / j$ for arbitrary $z \in(0,1)$. In the framework of statistical mechanics, $z$ can thus be viewed as

Received by the editors October 18th, 2016; accepted May 1st, 2017.

2010 Mathematics Subject Classification. 60C05, 60F05.

Key words and phrases. random permutation, total number of cycles, cycle weights, central limit theorem.

H.S. is supported by a PhD scholarship from Deutsche Telekom Stiftung. The authors gratefully acknowledge support by the DFG project BE 5267/1-1. 
the fugacity and the joint distribution of the $\tilde{c}_{j}$ as the grand canonical ensemble corresponding to the measures $\mathbb{P}_{n}$. Noting that the limit $n \rightarrow \infty$ corresponds to $z \rightarrow$ 1, Equation (1.1) is then easy to guess and not very hard to prove. Indeed, much more is true: DeLaurentis and Pittel (1985) show that for $C_{n, t}(\sigma)=\sum_{j=1}^{n^{t}} c_{j}(\sigma)$, the joint distribution of $\left((\log n)^{-1 / 2}\left(C_{n, t}-t \log n\right)\right)_{0 \leq t \leq 1}$ converges to standard Brownian motion on the time interval $[0,1]$. Gončarov (1962) and Kolčin (1971) show that the finite dimensional distributions of the sequence $\left(c_{j}\right)$ converge to independent Poisson variables $C_{j}$ with parameters $1 / j$. Arratia and Tavaré (1992) significantly improve this result: they show that the variation distance between the joint distribution of $\left(c_{j}\right)_{j \leq b(n)}$ and the independent Poisson variables $\left(C_{j}\right)_{j \leq b(n)}$ converges to zero if and only if $\lim _{n \rightarrow \infty} b(n) / n=0$; they even show the same result when considering a collection of $b(n)$ indices instead of the $b(n)$ first ones. So, the uniform measure on permutations is very well understood.

A natural next step is to study conditional measures, i.e. the uniform distribution on certain subsets of $\mathcal{S}_{n}$. We will be interested in permutations where only certain cycle lengths are allowed. Here, Theorem 3 in Arratia and Tavaré (1992) gives some interesting, partly more general answers: if we consider the uniform distribution on permutations such that for all $j$ from a subset $J \subset\{1, \ldots, b(n)\}$, the cycle counts $c_{j}$ have prescribed values $\bar{c}_{j}$, the variation distance between the remaining cycle counts and the corresponding independent Poisson variables $C_{j}$ still converges to zero as $n \rightarrow \infty$. In particular, we can condition $\bar{c}_{j}=0$ for as many $j \leq b(n)$ as we like and still get the Poisson convergence. However, the results of Arratia and Tavaré (1992) do not allow us to condition on $\bar{c}_{j}=0$ for $j>b(n)$, i.e. we cannot force large cycles to disappear. Conditioning on the absence of some large cycles is the content of the theory of $A$-permutations. Here, a set $A \subset \mathbb{N}$ is fixed, and the sequence of uniform measures on the sets of permutations $T_{n}(A)$ on $\{1, \ldots n\}$ and with cycle lengths in $A$ is studied. Under the condition that for each $n$ the number of permutations in $T_{n}(A)$ is large enough (precisely, of order $(n-1) ! n^{\alpha}$ for some $\alpha \in(0,1])$, Yakimiv shows the Poisson convergence of finite dimensional distributions Yakymiv (2007b) and the central limit theorem for the total number of cycles Yakymiv (2007a). The latter paper also contains a long list of references to the vast literature on $A$-permutations.

The situation is different when the set $A$ depends on $n$ and is such that long cycles are forbidden. For simplicity think of conditioning on the absence of cycles longer than $n^{\alpha}$ where $0<\alpha<1$. To see why this drastically changes the situation, recall that for large $n$ under the uniform measure on permutations, the fraction of indices in cycles of order $o(n)$ converges to zero. So, even though macroscopic (order $n$ ) cycles do not contribute significantly to the cycle count, they absorb the majority of indices. If we exclude them, the total number of cycles must grow much faster than logarithmically. Indeed, we will show below that when excluding cycles longer than $n^{\alpha}$, the average number of cycles grows like $n^{1-\alpha}$. Since all of the above mentioned methods in essence rely on the fact that the total cycle number is well approximated by a sum of independent Poisson random variables $\tilde{c}_{j}$ with parameters $1 / j$, they all predict a logarithmic growth of the cycle count. So none of them can possibly apply to the situation of restricted cycle lengths. It is thus interesting that the central limit theorem for the total number of cycles is robust enough to carry over to the case of permutations without long cycles. The proof of 
this statement (for rather general restrictions on cycle lengths) is the main result of the present paper.

A somewhat complementary result is obtained by Nikeghbali et al. (2013). They consider permutations of $n$ elements having only cycle lengths in a set $A_{n} \subset \mathbb{N}$ containing an 'interval' $\left\{a_{n}+1, a_{n}+2, \ldots, n\right\}$ with $a_{n}=o(n)$, i.e. $A_{n}$ contains all the 'long' cycles. They obtain a functional central limit theorem, again with logarithmic average cycle number. Indeed, they can treat slightly more general types of permutations by including cycle weights: the probability of a permutation $\sigma$ is modified by a Boltzmann factor $\exp \left(-\sum \alpha_{j} c_{j}(\sigma)\right)$, where the $\alpha_{j}$ correspond to the energies of cycles of length $j$. The case of constant $\alpha_{j}=\alpha$ corresponds to the Ewens distribution with parameter $\exp (\alpha)$, see Hansen (1990) for the first proof of a functional central limit theorem in that case.

A different use of cycle weights actually leads to the two only other situations that we are aware of where the typical number of cycles is not logarithmic and satisfies a central limit theorem. Firstly, Maples et al. (2012) consider the generalized Ewens measure with cycle weights of the form $\prod j^{\alpha c_{j}(\sigma)}$ with $\alpha>0$. Secondly, Bogachev and Zeindler (2015) replace the sequence $\alpha_{j}$ by a doubly indexed sequence $\alpha_{j, n}$. Under suitable assumptions on the asymptotics of the $\alpha_{j, n}$, they show (among many other things) that the number of cycles is of order $n$ and satisfies a central limit theorem. In particular, this implies that, contrary to the situation in the present paper, there is a positive fraction of points in finite cycles. The latter property motivates the name 'surrogate spatial random permutations' in the title of Bogachev and Zeindler (2015): while for random permutations it is in general rare to see a fraction of indices in finite cycles due to very strong entropic effects, this phenomenon becomes the norm when we add a spatial component to the model. These spatial random permutations are still relatively little understood, but appear to have many intriguing properties such as a phase transition from a regime without long cycles to one with a mixture of long and short cycles (proved for a special case in Betz and Ueltschi, 2011) and a rich geometry of the set of points in long cycles (see Grosskinsky et al., 2012; Betz, 2014 for some simulations). We refer the reader to Betz (2014) and the references therein for more information about these models.

\section{Results}

Let $\alpha: \mathbb{N} \rightarrow \mathbb{N}$, and consider

$$
S_{n, \alpha}:=\left\{\sigma \in S_{n}: \text { the cycles of } \sigma \text { are not longer than } \alpha(n)\right\},
$$

the set of permutations with $n$ elements and all cycles shorter than $\alpha(n)$. Let $\mathbb{P}_{n, \alpha}$ denote the uniform distribution on $S_{n, \alpha}$, and write

$$
C(\sigma)=\sum_{j=1}^{n} c_{j}(\sigma)
$$

for the sum of cycle counts $c_{j}(\sigma)$. For $\delta>0$ and all $w \in(1-\delta, 1+\delta)$, let $x_{n, \alpha}(w)$ be the unique positive solution of the equation

$$
\sum_{j=1}^{\alpha(n)} x_{n, \alpha}^{j}(w)=\frac{n}{w} .
$$


Throughout this paper, assumptions about $\alpha(n)$ and $\delta$ will be such that

$$
x_{n, \alpha}(w)>1
$$

holds for all $w$. Then, $x_{n, \alpha}$ is the unique root of the polynomial

$$
w x^{\alpha(n)+1}-(w+n) x+n
$$

that is contained in the interval $(1, \infty)$. Let us further write

$$
m_{n, \alpha}:=\sum_{j=1}^{\alpha(n)} \frac{x_{n, \alpha}^{j}(1)}{j}, \quad v_{n, \alpha}:=m_{n, \alpha}+n \frac{x_{n, \alpha}^{\prime}(1)}{x_{n, \alpha}(1)},
$$

where $x_{n, \alpha}^{\prime}$ denotes the derivative of $x_{n, \alpha}$. Our main result is

Theorem 2.1. Assume that

$$
\liminf _{n \rightarrow \infty} \alpha(n) \geq 4
$$

and

$$
\limsup _{n \rightarrow \infty} \frac{\alpha(n)}{n} \log (n)(\log (\log (n)))^{2}<\frac{1}{12 \pi^{2} \mathrm{e}} .
$$

Then for all $y \in \mathbb{R}$,

$$
\lim _{n \rightarrow \infty} \mathbb{P}_{n, \alpha}\left(C-m_{n, \alpha} \leq y \sqrt{v_{n, \alpha}}\right)=\Phi(y) .
$$

Here, $\Phi$ is the distribution function of the standard normal distribution.

Note that there is an extra term in the variance in equation (2.4). So in contrast to the classical situation, asymptotic mean and variance need not be of the same order. Under some additional assumptions on the function $\alpha$, we can derive asymptotic expressions for $m_{n, \alpha}$ and $v_{n, \alpha}$ and see that they are indeed of different order. For this, recall first the concept of asymptotic expansion. Let $\left(\phi_{k}\right)_{k \in \mathbb{N}}$ be a sequence of functions such that for all $k$

$$
\phi_{k+1}(z)=o\left(\phi_{k}(z)\right)
$$

as $z \rightarrow \infty$. We say that $\sum_{k=0}^{\infty} a_{k} \phi_{k}(z)$ is an asymptotic expansion for the function $f(z)$ as $z \rightarrow \infty$, and write

$$
f(z) \sim \sum_{k=0}^{\infty} a_{k} \phi_{k}(z)
$$

if for all $N \in \mathbb{N}$

$$
f(z)=\sum_{k=0}^{N} a_{k} \phi_{k}(z)+o\left(\phi_{N}(z)\right)
$$

as $z \rightarrow \infty$ (cf., e.g., Murray, 1984).

Let us write $\xi=\xi(u)$ for the non-zero solution of

$$
\exp (\xi)=1+u \xi
$$

with $\xi(1)=0$. Note that $\log (u)<\xi(u) \leq 2 \log (u)$ for $u>1$ (cf. Manstavičius and Petuchovas, 2016). 
Theorem 2.2. Assume that there are $\alpha_{0}, \alpha_{1} \in(0,1)$ with

$$
\liminf _{n \rightarrow \infty} n^{-\alpha_{0}} \alpha(n)=\infty \quad \text { and } \quad \limsup _{n \rightarrow \infty} n^{-\alpha_{1}} \alpha(n)=0 .
$$

Then the quantities defined in (2.4) have the asymptotic expansions

$$
m_{n, \alpha} \sim \frac{n}{\alpha(n)} \sum_{k=0}^{\infty} \frac{k !}{\left(\xi\left(\frac{n}{\alpha(n)}\right)\right)^{k}}
$$

and

$$
v_{n, \alpha} \sim \frac{n}{\alpha(n)} \sum_{k=2}^{\infty} \frac{k !-1}{\left(\xi\left(\frac{n}{\alpha(n)}\right)\right)^{k}} .
$$

This result shows that the average cycle count is close to its theoretical minimum, or in other words the average cycle lengths are close to maximal. This supports observations made e.g. in Betz et al. (2011) that 'entropy' is extremely strong in random permutations in the sense that it is very hard to 'force' many short cycles by modifying the uniform measure on permutations in natural ways, such as cycle weights or nontrivial conditioning. The theorem also suggests that most indices are contained in 'long' cycles. These questions will be addressed in a detailed manner in a forthcoming paper.

\section{Proofs: overview}

We will use the following criterion for a central limit theorem:

Proposition 3.1. Sachkov (1997) Let $A_{n}$ be a sequence of integer-valued random variables and assume that, as $n \rightarrow \infty$, their probability generating functions $H_{n}$ can be written as

$$
H_{n}(w)=\exp \left(h_{n}(w)\right)(1+o(1)),
$$

uniformly in the interval $W:=(1-\delta, 1+\delta)$ for some $\delta>0$, where $h_{n}$ has the following properties: $h_{n}$ is thrice continuously differentiable in $W$, and

$$
\lim _{n \rightarrow \infty} \sup _{w \in W} \frac{h_{n}^{\prime \prime \prime}(w)}{\left(h_{n}^{\prime}(1)+h_{n}^{\prime \prime}(1)\right)^{\frac{3}{2}}}=0 .
$$

Then the distribution of the random variable

$$
\eta_{n}:=\frac{A_{n}-h_{n}^{\prime}(1)}{\left(h_{n}^{\prime}(1)+h_{n}^{\prime \prime}(1)\right)^{\frac{1}{2}}}
$$

converges weakly to a standard normal distribution as $n \rightarrow \infty$.

For applying the above result, we need information on the generating function of the random cycle count $C$ under $\mathbb{P}_{n, \alpha}$. As is well known (see e.g. Flajolet and Sedgewick, 2009, Section II.4), the cardinality of $S_{n, \alpha}$ is given by

$$
\left|S_{n, \alpha}\right|=n !\left[z^{n}\right] \exp \left(\sum_{j=1}^{\alpha(n)} \frac{z^{j}}{j}\right)=n ! \frac{1}{2 \pi i} \int_{\gamma} \exp \left(\sum_{j=1}^{\alpha(n)} \frac{z^{j}}{j}\right) \frac{\mathrm{d} z}{z^{n+1}}
$$


where $\gamma$ is any closed curve around the origin in the complex plane. The probability generating function $H_{n, \alpha}(w)$ of $C$ under $\mathbb{P}_{n, \alpha}$ can therefore be written as

$$
H_{n, \alpha}(w)=c_{n, \alpha} \frac{1}{2 \pi i} \int_{\gamma} \exp \left(w \sum_{j=1}^{\alpha(n)} \frac{z^{j}}{j}\right) \frac{\mathrm{d} z}{z^{n+1}}
$$

where $c_{n, \alpha}$ is a normalization constant (which does not depend on $w$ ) (cf. Flajolet and Sedgewick, 2009, Section III.4).

In Sachkov (1997), Proposition 3.1 is applied to cases such as Equation (3.3), and an approximation using the saddle point method is given. The difference with our situation however is that in the cited reference, there are fixed polynomials in the exponential, while in our case the upper limit $\alpha(n)$ of the sum will typically grow with $n$. To handle this situation, we use a slight generalization of precise asymptotic estimates recently derived by Manstavičius and Petuchovas $(2015,2016)$.

Proposition 3.2. Under the assumptions of Theorem 2.1, the following asymptotic relation holds as $n \rightarrow \infty$, uniformly for $w \in(1-\delta, 1+\delta)$ :

$$
H_{n, \alpha}(w)=c_{n, \alpha} \frac{\exp \left(w \sum_{j=1}^{\alpha(n)} \frac{x_{n, \alpha}^{j}(w)}{j}\right)}{x_{n, \alpha}^{n}(w) \sqrt{2 \pi w \sum_{j=1}^{\alpha(n)} j x_{n, \alpha}^{j}(w)}}\left(1+O\left(\frac{\alpha(n)}{n}\right)\right) .
$$

Here, $x_{n, \alpha}(w)$ is the unique positive solution of (2.1).

Proof: First note that because of the strict inequality in (2.5), there exists $\delta>0$ such that for all $w \in(1-\delta, 1+\delta)$ and all large enough $n$, we have

$$
\alpha(n) \leq \frac{1}{12 \pi^{2} \mathrm{e}} \frac{n}{w \log (n)(\log (\log (n)))^{2}} .
$$

The proposition is then an adaptation of Theorem 2 in Manstavičius and Petuchovas (2016). The latter makes use of the saddle point method and treats the case $w=$ 1. The proof given by Manstavičius and Petuchovas extends almost verbatim to the present situation when only large $n$ are considered. The main difference is a maximization of the resulting constants in the error terms with respect to $w \in$ $(1-\delta, 1+\delta)$, which does not affect their asymptotics.

Remark 3.3. Indeed, Manstavičius and Petuchovas (2016) proves the asymptotics in the case $w=1$ without restrictions on $\alpha$, but the generalization to $w \neq 1$ is not as obvious without the growth restriction (2.5), so we stick with it.

To connect Propositions 3.1 and 3.2 and prove the theorem, we thus need asymptotics of the derivatives of

$$
\begin{aligned}
h_{n, \alpha}(w)= & \log \left(c_{n, \alpha}\right)+w \sum_{j=1}^{\alpha(n)} \frac{x_{n, \alpha}^{j}(w)}{j} \\
& -n \log \left(x_{n, \alpha}(w)\right)-\frac{1}{2} \log \left(2 \pi w \sum_{j=1}^{\alpha(n)} j x_{n, \alpha}^{j}(w)\right) .
\end{aligned}
$$

This is done in Lemmas 4.1 to 4.6 , where it is proved that

$$
h_{n, \alpha}^{\prime}(w)=\sum_{j=1}^{\alpha(n)} \frac{x_{n, \alpha}^{j}(w)}{j}+O(1), \quad h_{n, \alpha}^{\prime \prime}(w)=\frac{n x_{n, \alpha}^{\prime}(w)}{w x_{n, \alpha}(w)}+O(1),
$$


and that $h_{n, \alpha}^{\prime \prime \prime}(w)=O(n / \alpha(n))$. Since it turns out (see Lemma 4.7 and the discussion thereafter) that the leading terms of both $h_{n, \alpha}^{\prime}$ and $h_{n, \alpha}^{\prime \prime}$ behave like $n / \alpha(n)$ for large $n$ but have different signs, a very fine asymptotic analysis is necessary to ensure that (3.1) holds. This is done in Propositions 4.8 and 4.9. After this, Proposition 3.1 can be used to prove Theorem 2.1.

The proof of Theorem 2.2 refines the results of Propositions 4.8 and 4.9 by adding information on the asymptotics of $x_{n, \alpha}(1)$. These are given in Lemma 4.11, and are essentially due to Manstavičius and Petuchovas (2016).

\section{Proofs: details}

The following lemma presents an expression for the first derivative of $h_{n, \alpha}$.

Lemma 4.1. Let $\alpha: \mathbb{N} \rightarrow \mathbb{N}$ and $0<\delta<1$. Then the following relation holds for all $n \in \mathbb{N}$ and $w \in(1-\delta, 1+\delta)$ :

$$
h_{n, \alpha}^{\prime}(w)=\sum_{j=1}^{\alpha(n)} \frac{x_{n, \alpha}^{j}(w)}{j}-\frac{1}{2} \frac{x_{n, \alpha}^{\prime}(w)}{x_{n, \alpha}(w)}+\frac{1}{2} \frac{x_{n, \alpha}^{\prime \prime}(w)}{x_{n, \alpha}^{\prime}(w)}+\frac{1}{2 w} .
$$

Proof: Let $f_{n}(x):=\sum_{j=1}^{\alpha(n)} x^{j}$. Then $f_{n}$ is strictly increasing on $\mathbb{R}_{+}$and $f_{n}^{\prime}(x)>0$ for all $x>0$. According to Equation (2.1), the saddle point is given by

$$
x_{n, \alpha}(w)=f_{n}^{-1}\left(\frac{n}{w}\right) .
$$

Hence, $x_{n, \alpha}$ is differentiable in $w$ by the inverse mapping theorem and

$$
x_{n, \alpha}^{\prime}(w)=-\frac{1}{f_{n}^{\prime}\left(x_{n, \alpha}(w)\right)} \frac{n}{w^{2}} .
$$

A bootstrapping argument shows that $x_{n, \alpha}$ is infinitely often differentiable. Due to Equation (2.1), the derivative of the first three terms of Equation (3.4) is the first term of Equation (4.1). For the last term of Equation (3.4),

$$
-\frac{1}{2} \log \left(2 \pi w \sum_{j=1}^{\alpha(n)} j\left(x_{n, \alpha}(w)\right)^{j}\right)
$$

use the identity

$$
\sum_{j=1}^{\alpha(n)} j\left(x_{n, \alpha}(w)\right)^{j}=-n \frac{x_{n, \alpha}(w)}{x_{n, \alpha}^{\prime}(w)} \frac{1}{w^{2}}
$$

obtained by differentiating Equation (2.1). Then compute the derivative of the resulting term.

The ingredients necessary for the proof of the theorem are asymptotics for the derivatives of $h_{n, \alpha}$. A first step towards this goal is

Lemma 4.2. Let $\alpha: \mathbb{N} \rightarrow \mathbb{N}$ and $0<\delta<1$ such that $\frac{n}{w \alpha(n)}>1$ for large $n$ and $w \in(1-\delta, 1+\delta)$. Then

$$
\lim _{n \rightarrow \infty} \alpha(n) \log \left(x_{n, \alpha}(w)\right)=\infty
$$

if and only if

$$
\lim _{n \rightarrow \infty} \frac{\alpha(n)}{n}=0
$$


Proof: Manstavičius and Petuchovas (2016) Comparing geometric and arithmetic means yields

$$
\left(x_{n, \alpha}(w)\right)^{\frac{\alpha(n)+1}{2}}=\left(\prod_{j=1}^{\alpha(n)}\left(x_{n, \alpha}(w)\right)^{j}\right)^{\frac{1}{\alpha(n)}} \leq \frac{1}{\alpha(n)} \sum_{j=1}^{\alpha(n)}\left(x_{n, \alpha}(w)\right)^{j}=\frac{n}{w \alpha(n)} .
$$

From Equation (2.1),

$$
\left(x_{n, \alpha}(w)\right)^{\alpha(n)} \geq \frac{n}{w \alpha(n)}
$$

Hence,

$$
\left(x_{n, \alpha}(w)\right)^{\frac{\alpha(n)+1}{2}} \leq \frac{n}{w \alpha(n)} \leq\left(x_{n, \alpha}(w)\right)^{\alpha(n)}
$$

and, since the logarithm is increasing and $\log \left(x_{n, \alpha}(w)\right) \geq 0$ by Equation (2.2),

$$
\frac{1}{2} \alpha(n) \log \left(x_{n, \alpha}(w)\right) \leq \log \left(\frac{n}{w \alpha(n)}\right) \leq \alpha(n) \log \left(x_{n, \alpha}(w)\right) .
$$

The claim is a direct consequence.

Remark 4.3. More detailed asymptotics for $x_{n, \alpha}(w)$ and related quantities can be found in Manstavičius and Petuchovas (2016).

Lemma 4.4. Let $\alpha: \mathbb{N} \rightarrow \mathbb{N}$ and $0<\delta<1$ such that $\frac{n}{w \alpha(n)}>1$ for large $n$ and $w \in(1-\delta, 1+\delta)$. Moreover, let

$$
\lim _{n \rightarrow \infty} \frac{\alpha(n)}{n}=0
$$

Then,

$$
\lim _{n \rightarrow \infty} \alpha(n) \frac{x_{n, \alpha}^{\prime}(w)}{x_{n, \alpha}(w)}=-\frac{1}{w}
$$

Proof: Starting from Equation (2.3), we have

$$
w\left[\left(x_{n, \alpha}(w)\right)^{\alpha(n)}-1\right]=n\left(1-\frac{1}{x_{n, \alpha}(w)}\right) .
$$

Differentiating Equation (4.5) with respect to $w$, solving for $x_{n, \alpha}^{\prime}(w)$, and applying once more Equation (2.3) yields

$$
x_{n, \alpha}^{\prime}(w)=\frac{\left(x_{n, \alpha}(w)-1\right) x_{n, \alpha}(w)}{w\left(1-\alpha(n)\left(x_{n, \alpha}(w)-1\right)-\frac{w \alpha(n) x_{n, \alpha}(w)}{n}\right)} .
$$

Hence,

$$
\begin{aligned}
\lim _{n \rightarrow \infty} \frac{x_{n, \alpha}(w)}{\alpha(n) x_{n, \alpha}^{\prime}(w)} & =\lim _{n \rightarrow \infty}\left[\frac{w}{\alpha(n)\left(x_{n, \alpha}(w)-1\right)}-w-\frac{w^{2} x_{n, \alpha}(w)}{n\left(x_{n, \alpha}(w)-1\right)}\right] \\
& =-w
\end{aligned}
$$

by

$$
x_{n, \alpha}(w)-1 \geq \log \left(x_{n, \alpha}(w)\right)
$$

(which follows from Equation (2.2)) and Lemma 4.2. 
Lemma 4.5. Let $\alpha: \mathbb{N} \rightarrow \mathbb{N}$ and $0<\delta<1$ as in Lemma 4.4. Then the following relations hold uniformly in $w \in(1-\delta, 1+\delta)$ as $n \rightarrow \infty$ :

$$
\begin{aligned}
& h_{n, \alpha}^{\prime}(w)=\sum_{j=1}^{\alpha(n)} \frac{x_{n, \alpha}^{j}(w)}{j}+O(1), \\
& h_{n, \alpha}^{\prime \prime}(w)=\frac{n x_{n, \alpha}^{\prime}(w)}{w x_{n, \alpha}(w)}+O(1),
\end{aligned}
$$

and

$$
h_{n, \alpha}^{\prime \prime \prime}(w)=\frac{n x_{n, \alpha}^{\prime \prime}(w)}{w x_{n, \alpha}(w)}-\frac{n x_{n, \alpha}^{\prime}(w)}{w^{2} x_{n, \alpha}(w)}-\frac{n}{w}\left(\frac{x_{n, \alpha}^{\prime}(w)}{x_{n, \alpha}(w)}\right)^{2}+O(1) .
$$

Proof: Let

$$
J_{k}(x):=\sum_{j=1}^{\alpha(n)} j^{k} x^{j}
$$

From Equation (4.2) we deduce

$$
\frac{x_{n, \alpha}^{\prime}(w)}{x_{n, \alpha}(w)} J_{1}\left(x_{n, \alpha}(w)\right)=-\frac{n}{w^{2}} .
$$

Together with Lemma 4.4, we conclude

$$
\lim _{n \rightarrow \infty} \frac{J_{1}\left(x_{n, \alpha}(w)\right)}{n \alpha(n)}=\frac{1}{w} .
$$

Moreover,

$$
J_{k}\left(x_{n, \alpha}(w)\right) \leq(\alpha(n))^{k-1} J_{1}\left(x_{n, \alpha}(w)\right)
$$

for all $k \geq 1$. Differentiating Equation (4.2) and applying Equation (4.10) yield

$$
\frac{x_{n, \alpha}^{\prime \prime}(w)}{x_{n, \alpha}(w)} J_{1}\left(x_{n, \alpha}(w)\right)+\frac{n}{w^{2}} \frac{x_{n, \alpha}^{\prime}(w)}{x_{n, \alpha}(w)}+\left(\frac{x_{n, \alpha}^{\prime}(w)}{x_{n, \alpha}(w)}\right)^{2} J_{2}\left(x_{n, \alpha}(w)\right)=\frac{2 n}{w^{3}} .
$$

Hence,

$$
\frac{x_{n, \alpha}^{\prime \prime}(w)}{x_{n, \alpha}(w)}=O\left(\frac{1}{\alpha(n)}\right)
$$

uniformly in $w \in(1-\delta, 1+\delta)$ by Lemma 4.4. Iterating this procedure, we obtain

$$
\frac{x_{n, \alpha}^{\prime \prime \prime}(w)}{x_{n, \alpha}(w)}=O\left(\frac{1}{\alpha(n)}\right)
$$

and

$$
\frac{x_{n, \alpha}^{(4)}(w)}{x_{n, \alpha}(w)}=O\left(\frac{1}{\alpha(n)}\right) .
$$

Equation (4.7) is now a direct consequence. Since it is easily shown that the derivatives of

and

$$
\frac{x_{n, \alpha}^{\prime}(w)}{x_{n, \alpha}(w)}
$$

$$
\frac{x_{n, \alpha}^{\prime \prime}(w)}{x_{n, \alpha}^{\prime}(w)}
$$

are also $O(1)$, Equation (4.8) follows from Equations (4.7) and (2.1). Further differentiation yields Equation (4.9). 
Lemma 4.6. Under the assumptions of Lemma 4.4,

$$
h_{n, \alpha}^{\prime \prime \prime}(w)=O\left(\frac{n}{\alpha(n)}\right)
$$

uniformly in $w \in(1-\delta, 1+\delta)$ as $n \rightarrow \infty$.

Proof: After the work done in the previous lemmata, this is an easy corollary. The dominating terms in Equation (4.9) have the required property.

Lemma 4.7. Under the assumptions of Lemma 4.4,

$$
\lim _{n \rightarrow \infty} \frac{\alpha(n)}{n} h_{n, \alpha}^{\prime \prime}(1)=-1 .
$$

Proof: The lemma follows from Equation (4.8) and Lemma 4.4.

The asymptotic behaviour of $h_{n, \alpha}^{\prime}(1)$ is determined by

$$
\begin{aligned}
\sum_{j=1}^{\alpha(n)} \frac{x_{n, \alpha}^{j}(1)}{j} & \geq \frac{1}{\alpha(n)} \sum_{j=1}^{\alpha(n)} x_{n, \alpha}^{j}(1) \\
& =\frac{n}{\alpha(n)}
\end{aligned}
$$

which competes with $-\frac{n}{\alpha(n)}$ due to the different sign. If the third derivative of $h_{n, \alpha}$ is to be dominated by

$$
\left(h_{n, \alpha}^{\prime}(1)+h_{n, \alpha}^{\prime \prime}(1)\right)^{\frac{3}{2}},
$$

more careful estimates are needed. Such estimates will be provided by Propositions 4.8 and 4.9 .

The exponential integral (DLMF, Equation (6.2.5)) is given by

$$
\operatorname{Ei}(x):=\text { p.v. } \int_{-\infty}^{x} \frac{\exp (t)}{t} \mathrm{~d} t
$$

and has the asymptotic expansion

$$
\operatorname{Ei}(x) \sim \frac{\exp (x)}{x} \sum_{k=0}^{\infty} \frac{k !}{x^{k}}
$$

as $x \rightarrow \infty$ (DLMF, Equation (6.12.2)).

Proposition 4.8. Let $\alpha: \mathbb{N} \rightarrow \mathbb{N}$ such that

$$
\lim _{n \rightarrow \infty} \frac{\alpha(n)}{n}=0
$$

and $\alpha(n) \geq 2$ for large $n$. Then

$$
\begin{aligned}
h_{n, \alpha}^{\prime}(1)= & \log (\alpha(n))+\frac{n}{\alpha(n)}+\frac{n}{(\alpha(n))^{2}\left(x_{n, \alpha}(1)-1\right)} \\
& +2 \frac{n}{(\alpha(n))^{3}\left(x_{n, \alpha}(1)-1\right) \log \left(x_{n, \alpha}(1)\right)} \\
& +p_{\alpha}(n)+O\left(\frac{n}{(\alpha(n))^{4}\left(\log \left(x_{n, \alpha}(1)\right)\right)^{2}\left(x_{n, \alpha}(1)-1\right)}\right) .
\end{aligned}
$$

Here, $p_{\alpha}$ is a non-negative function satisfying $p_{\alpha}(n)=O\left(\frac{\left(x_{n, \alpha}(1)\right)^{\alpha(n)}}{(\alpha(n))^{3} \log \left(x_{n, \alpha}(1)\right)}\right)$. 
Proof: We start by investigating the explicit term in Equation (4.7).

$$
\begin{aligned}
\sum_{j=1}^{\alpha(n)} \frac{x_{n, \alpha}^{j}(1)}{j} & =\sum_{j=1}^{\alpha(n)} \frac{1}{j}+\int_{1}^{x_{n, \alpha}(1)} \sum_{j=1}^{\alpha(n)} t^{j-1} \mathrm{~d} t \\
& =\sum_{j=1}^{\alpha(n)} \frac{1}{j}+\int_{0}^{\log \left(x_{n, \alpha}(1)\right)} \frac{\exp (\alpha(n) v)-1}{v} \frac{v \exp (v) \mathrm{d} v}{\exp (v)-1} .
\end{aligned}
$$

The second line follows from

$$
\sum_{j=1}^{\alpha(n)} t^{j-1}=\frac{t^{\alpha(n)}-1}{t-1}
$$

and the substitution $v=\log (t)$ (Manstavičius and Petuchovas, 2016). It is a wellknown fact about the harmonic numbers that

$$
\sum_{j=1}^{\alpha(n)} \frac{1}{j}=\log (\alpha(n))+O(1) .
$$

Let

$$
g(v):=\frac{v \exp (v)}{\exp (v)-1} .
$$

Then

$$
g^{\prime}(v)=\frac{\exp (v)[\exp (v)-1-v]}{(\exp (v)-1)^{2}}
$$

and

$$
g^{\prime \prime}(v)=\frac{\exp (v)[v \exp (v)-2 \exp (v)+v+2]}{(\exp (v)-1)^{3}} .
$$

Expanding the exponential functions in the numerator of $g^{\prime \prime}$ shows that

$$
g^{\prime \prime}(v) \geq 0
$$

for all $v \geq 0$. Moreover, $g^{\prime \prime}$ is bounded on $\mathbb{R}_{+}$since $\lim _{v \rightarrow 0} g^{\prime \prime}(v)=\frac{1}{6}$ and $\lim _{v \rightarrow \infty} g^{\prime \prime}(v)=0$.

By expanding $g$ about $\log \left(x_{n, \alpha}(1)\right)$, the integrand in Equation (4.14) becomes

$$
\begin{aligned}
& \frac{\exp (\alpha(n) v)-1}{v} \frac{v \exp (v)}{\exp (v)-1} \\
= & \frac{\log \left(x_{n, \alpha}(1)\right)}{1-\left(x_{n, \alpha}(1)\right)^{-1}} \frac{\exp (\alpha(n) v)-1}{v} \\
& +\frac{\left(x_{n, \alpha}(1)-1\right)-\log \left(x_{n, \alpha}(1)\right)}{\left(x_{n, \alpha}(1)-1\right)\left(1-\left(x_{n, \alpha}(1)\right)^{-1}\right)} \frac{\exp (\alpha(n) v)-1}{v}\left(v-\log \left(x_{n, \alpha}(1)\right)\right) \\
& +\frac{g^{\prime \prime}(\Xi(v))}{2} \frac{\exp (\alpha(n) v)-1}{v}\left(v-\log \left(x_{n, \alpha}(1)\right)\right)^{2} \\
= & \frac{1}{\left(1-\left(x_{n, \alpha}(1)\right)^{-1}\right)}\left[1-\frac{\log \left(x_{n, \alpha}(1)\right)}{x_{n, \alpha}(1)-1}\right](\exp (\alpha(n) v)-1)
\end{aligned}
$$




$$
\begin{aligned}
& +\frac{\left(\log \left(x_{n, \alpha}(1)\right)\right)^{2}}{\left(x_{n, \alpha}(1)-1\right)\left(1-\left(x_{n, \alpha}(1)\right)^{-1}\right)} \frac{\exp (\alpha(n) v)-1}{v} \\
& +\frac{g^{\prime \prime}(\Xi(v))}{2} \frac{\exp (\alpha(n) v)-1}{v}\left(v-\log \left(x_{n, \alpha}(1)\right)\right)^{2}
\end{aligned}
$$

where $0 \leq \Xi(v) \leq v$ by Taylor's theorem. An easy calculation yields

$$
\begin{aligned}
& \int_{0}^{\log \left(x_{n, \alpha}(1)\right)}(\exp (\alpha(n) v)-1) \mathrm{d} v \\
= & \frac{\left(x_{n, \alpha}(1)\right)^{\alpha(n)}}{\alpha(n)}-\log \left(x_{n, \alpha}(1)\right)-\frac{1}{\alpha(n)}
\end{aligned}
$$

and, by substituting $s=\alpha(n) v$ and applying Equations (4.11) and (4.12),

$$
\begin{aligned}
& \int_{0}^{\log \left(x_{n, \alpha}(1)\right)} \frac{\exp (\alpha(n) v)-1}{v} \mathrm{~d} v \\
= & \int_{0}^{1} \frac{\exp (s)-1}{s} \mathrm{~d} s+\int_{1}^{\alpha(n) \log \left(x_{n, \alpha}(1)\right)} \frac{\exp (s)}{s} \mathrm{~d} s-\int_{1}^{\alpha(n) \log \left(x_{n, \alpha}(1)\right)} \frac{\mathrm{d} s}{s} \\
& +\operatorname{Ei}(1)-\operatorname{Ei}(1) \\
= & \operatorname{Ei}\left(\alpha(n) \log \left(x_{n, \alpha}(1)\right)\right)-\log \log \left(\left(x_{n, \alpha}(1)\right)^{\alpha(n)}\right)+O(1) \\
= & \frac{\left(x_{n, \alpha}(1)\right)^{\alpha(n)}}{\alpha(n) \log \left(x_{n, \alpha}(1)\right)}\left[1+\frac{1}{\alpha(n) \log \left(x_{n, \alpha}(1)\right)}+\frac{2}{\left(\alpha(n) \log \left(x_{n, \alpha}(1)\right)^{2}\right)}\right] \\
& +O\left(\frac{\left(x_{n, \alpha}(1)\right)^{\alpha(n)}}{\alpha(n) \log \left(x_{n, \alpha}(1)\right)} \frac{1}{\left(\alpha(n) \log \left(x_{n, \alpha}(1)\right)\right)^{3}}\right) .
\end{aligned}
$$

Due to Equations (2.3) and (4.15),

$$
\begin{aligned}
\left(x_{n, \alpha}(1)\right)^{\alpha(n)} & =1+n\left(1-\left(x_{n, \alpha}(1)\right)^{-1}\right) \\
& \sim n\left(1-\left(x_{n, \alpha}(1)\right)^{-1}\right) .
\end{aligned}
$$

Applying Equations (4.17), (4.18), and (4.19) to the integral in Equation (4.14), we have

$$
\begin{aligned}
& \int_{0}^{\log \left(x_{n, \alpha}(1)\right)} \frac{\exp (\alpha(n) v)-1}{v} \frac{v \exp (v)}{\exp (v)-1} \mathrm{~d} v \\
= & \frac{n}{\alpha(n)}+\frac{n}{(\alpha(n))^{2}\left(x_{n, \alpha}(1)-1\right)}+2 \frac{n}{(\alpha(n))^{3}\left(x_{n, \alpha}(1)-1\right) \log \left(x_{n, \alpha}(1)\right)} \\
& +p_{\alpha}(n)+O\left(\frac{n}{(\alpha(n))^{4}\left(\log \left(x_{n, \alpha}(1)\right)\right)^{2}\left(x_{n, \alpha}(1)-1\right)}\right)
\end{aligned}
$$

with

$$
p_{\alpha}(n):=\int_{0}^{\log \left(x_{n, \alpha}(1)\right)} \frac{g^{\prime \prime}(\Xi(v))}{2} \frac{\exp (\alpha(n) v)-1}{v}\left(v-\log \left(x_{n, \alpha}(1)\right)\right)^{2} \mathrm{~d} v \geq 0 .
$$


Since $g^{\prime \prime}$ is bounded, a calculation similar to Equations (4.17) and (4.18) yields

$$
p_{\alpha}(n)=O\left(\frac{\left(x_{n, \alpha}(1)\right)^{\alpha(n)}}{(\alpha(n))^{3} \log \left(x_{n, \alpha}(1)\right)}\right) .
$$

Thus, $\sum_{j=1}^{\alpha(n)} \frac{x_{n, \alpha}^{j}(1)}{j}$ equals the right-hand side of Equation (4.13). It now remains to be shown that

$$
O(1) \subset O\left(\frac{n}{(\alpha(n))^{4}\left(\log \left(x_{n, \alpha}(1)\right)\right)^{2}\left(x_{n, \alpha}(1)-1\right)}\right) .
$$

For this to hold, $\alpha(n)\left(x_{n, \alpha}(1)-1\right)$ needs to be bounded from above by suitable terms. We have to consider two cases. If $\alpha(n) \geq \log (n)$, then

$$
\begin{aligned}
\alpha(n)\left(x_{n, \alpha}(1)-1\right) & =\alpha(n)\left[\exp \left(\log \left(x_{n, \alpha}(1)\right)\right)-1\right] \\
& \leq \alpha(n)\left[\exp \left(2 \frac{\log \left(\frac{n}{\alpha(n)}\right)}{\alpha(n)}\right)-1\right] \\
& =O\left(\log \left(\frac{n}{\alpha(n)}\right)\right)
\end{aligned}
$$

by Equation (4.4). If $2 \leq \alpha(n)<\log (n)$, we use

$$
\alpha(n)\left(x_{n, \alpha}(1)-1\right) \leq \alpha(n) n^{\frac{1}{\alpha(n)}}
$$

by Equation (2.1).

Proposition 4.9. Let $\alpha: \mathbb{N} \rightarrow \mathbb{N}$ such that

$$
\lim _{n \rightarrow \infty} \frac{\alpha(n)}{n}=0
$$

Then

$$
h_{n, \alpha}^{\prime \prime}(1)=-\frac{n}{\alpha(n)} \sum_{j=0}^{\infty}\left(\frac{1}{\alpha(n)\left(x_{n, \alpha}(1)-1\right)+\frac{\alpha(n) x_{n, \alpha}(1)}{n}}\right)^{j}+O(1)
$$

as $n \rightarrow \infty$.

If also $\alpha(n) \geq 2$ for large $n$, then

$$
\begin{aligned}
h_{n, \alpha}^{\prime \prime}(1)= & -\frac{n}{\alpha(n)} \sum_{j=0}^{2}\left(\frac{1}{\alpha(n)\left(x_{n, \alpha}(1)-1\right)}\right)^{j} \\
& +o\left(\frac{n}{(\alpha(n))^{3}\left(x_{n, \alpha}(1)-1\right)^{2}}\right)+O(1) .
\end{aligned}
$$

Proof: According to Lemma 4.5 and Equation (4.6),

$$
\begin{aligned}
h_{n, \alpha}^{\prime \prime}(1) & =\frac{n x_{n, \alpha}^{\prime}(1)}{x_{n, \alpha}(1)}+O(1) \\
& =\frac{n\left(x_{n, \alpha}(1)-1\right)}{1-\alpha(n)\left(x_{n, \alpha}(1)-1\right)-\frac{\alpha(n) x_{n, \alpha}(1)}{n}}+O(1)
\end{aligned}
$$




$$
\begin{aligned}
= & \frac{n}{\alpha(n)} \frac{1}{\frac{1}{\alpha(n)\left(x_{n, \alpha}(1)-1\right)+\frac{\alpha(n) x_{n, \alpha}(1)}{n}}-1} \\
& -\frac{x_{n, \alpha}(1)}{1-\left(\alpha(n)\left(x_{n, \alpha}(1)-1\right)+\frac{\alpha(n) x_{n, \alpha}(1)}{n}\right)}+O(1) \\
= & -\frac{n}{\alpha(n)} \sum_{j=0}^{\infty}\left(\frac{1}{\alpha(n)\left(x_{n, \alpha}(1)-1\right)+\frac{\alpha(n) x_{n, \alpha}(1)}{n}}\right)^{j}+O(1) .
\end{aligned}
$$

The last line applies the geometric series and

$$
\frac{x_{n, \alpha}(1)}{1-\left(\alpha(n)\left(x_{n, \alpha}(1)-1\right)+\frac{\alpha(n) x_{n, \alpha}(1)}{n}\right)}=O(1),
$$

which follows easily from Lemma 4.2.

From

$$
\begin{aligned}
& \frac{1}{\alpha(n)\left(x_{n, \alpha}(1)-1\right)+\frac{\alpha(n) x_{n, \alpha}(1)}{n}} \\
= & \frac{1}{\alpha(n)\left(x_{n, \alpha}(1)-1\right)} \\
& -\frac{\alpha(n)}{n} \frac{x_{n, \alpha}(1)}{(\alpha(n))^{2}\left(x_{n, \alpha}(1)-1\right)^{2}+\frac{(\alpha(n))^{2}}{n} x_{n, \alpha}(1)\left(x_{n, \alpha}(1)-1\right)}
\end{aligned}
$$

and

$$
x_{n, \alpha}(1) \leq\left(\frac{n}{\alpha(n)}\right)^{\frac{2}{1+\alpha(n)}}
$$

by Equation (4.3), one may conclude that

$$
\begin{aligned}
& \frac{\alpha(n)}{n} \frac{x_{n, \alpha}(1)}{(\alpha(n))^{2}\left(x_{n, \alpha}(1)-1\right)^{2}+\frac{(\alpha(n))^{2}}{n} x_{n, \alpha}(1)\left(x_{n, \alpha}(1)-1\right)} \\
= & o\left(\left(\frac{1}{\alpha(n)\left(x_{n, \alpha}(1)-1\right)}\right)^{2}\right)
\end{aligned}
$$

if $\alpha(n) \geq 2$. The second claim then follows.

The tools needed to prove Theorem 2.1 are now available.

Proof of Theorem 2.1: By Equation (2.5), $\alpha(n) / n \rightarrow 0$ as $n \rightarrow \infty$. Propositions 4.8 and 4.9 yield

$$
\begin{aligned}
& h_{n, \alpha}^{\prime}(1)+h_{n, \alpha}^{\prime \prime}(1) \\
= & \log (\alpha(n))+\frac{n}{\alpha(n)}+\frac{n}{(\alpha(n))^{2}\left(x_{n, \alpha}(1)-1\right)} \\
& +2 \frac{n}{(\alpha(n))^{3}\left(x_{n, \alpha}(1)-1\right) \log \left(x_{n, \alpha}(1)\right)}-\frac{n}{\alpha(n)} \sum_{j=0}^{2}\left(\frac{1}{\alpha(n)\left(x_{n, \alpha}(1)-1\right)}\right)^{j} \\
& +p_{\alpha}(n)+O\left(\frac{n}{(\alpha(n))^{4}\left(\log \left(x_{n, \alpha}(1)\right)\right)^{2}\left(x_{n, \alpha}(1)-1\right)}\right)
\end{aligned}
$$




$$
\begin{aligned}
& \quad+o\left(\frac{n}{(\alpha(n))^{3}\left(x_{n, \alpha}(1)-1\right)^{2}}\right) \\
& =\log (\alpha(n))+2 \frac{n}{(\alpha(n))^{3}\left(x_{n, \alpha}(1)-1\right) \log \left(x_{n, \alpha}(1)\right)}-\frac{n}{(\alpha(n))^{3}\left(x_{n, \alpha}(1)-1\right)^{2}} \\
& +p_{\alpha}(n)+O\left(\frac{n}{(\alpha(n))^{4}\left(\log \left(x_{n, \alpha}(1)\right)\right)^{2}\left(x_{n, \alpha}(1)-1\right)}\right) \\
& +o\left(\frac{n}{(\alpha(n))^{3}\left(x_{n, \alpha}(1)-1\right)^{2}}\right) .
\end{aligned}
$$

Since

$$
x-1 \geq \log (x)
$$

for $x>1$, we have

$$
\lim _{n \rightarrow \infty} \frac{h_{n, \alpha}^{\prime}(1)+h_{n, \alpha}^{\prime \prime}(1)}{\frac{n}{(\alpha(n))^{3}\left(x_{n, \alpha}(1)-1\right) \log \left(x_{n, \alpha}(1)\right)}} \geq 1
$$

by Lemma 4.2. By Lemma 4.6 and Equations (4.4), (4.21), and (4.22) as well as $\alpha(n) \geq 4$,

uniformly in $w$.

$$
\lim _{n \rightarrow \infty} \frac{h_{n, \alpha}^{\prime \prime \prime}(w)}{\left(\frac{n}{(\alpha(n))^{3}\left(x_{n, \alpha}(1)-1\right) \log \left(x_{n, \alpha}(1)\right)}\right)^{\frac{3}{2}}}=0
$$

Therefore, Proposition 3.1 may be applied and the theorem is proved.

Remark 4.10. Equations (4.13) and (4.23) show that the behavior of $m_{n, \alpha}$ and $v_{n, \alpha}$ changes when $\log (\alpha(n))$ surpasses $\frac{n}{\alpha(n)}$ and becomes the dominating term. This blends in nicely with the fact that the classical uniform model has asymptotic expectation and variance of $\log (n)$.

Recall that the function $\xi(u)$ is defined as the non-zero solution of $\exp (\xi(u))=$ $1+u \xi(u)$ for $u>1$ by Equation (2.6).

Lemma 4.11. Manstavičius and Petuchovas (2016) Let $\alpha: \mathbb{N} \rightarrow \mathbb{N}$ such that $\log (n) \leq \alpha(n)<n$ for all $n$. Then

$$
\begin{gathered}
x_{n, \alpha}(1)=\exp \left(\frac{\xi\left(\frac{n}{\alpha(n)}\right)}{\alpha(n)}\right)+O\left(\frac{\log \left(\frac{n}{\alpha(n)}+1\right)}{\alpha(n))^{2}}\right), \\
\xi\left(\frac{n}{\alpha(n)}\right)=\log \left(\frac{n}{\alpha(n)}\right)+\log \left(\log \left(\frac{n}{\alpha(n)}+2\right)\right)+O\left(\frac{\log \left(\log \left(\frac{n}{\alpha(n)}+2\right)\right)}{\log \left(\frac{n}{\alpha(n)}+2\right)}\right)
\end{gathered}
$$

and

$$
\alpha(n) \log \left(x_{n, \alpha}(1)\right)=\xi\left(\frac{n}{\alpha(n)}\right)+O\left(\frac{\log \left(\frac{n}{\alpha(n)}+1\right)}{\alpha(n)}\right)
$$

as $n \rightarrow \infty$. 
Proof: The first and second parts are reformulations of assertions in Lemmata 10 and 6 in Manstavičius and Petuchovas (2016) from which the third part follows. The third equation has already been stated in an earlier version of Manstavičius and Petuchovas (2016), albeit for a smaller range of possible $\alpha$.

Lemma 4.12. Manstavičius and Petuchovas (2016) Let

$$
T_{K}(z):=\int_{0}^{z} \frac{\exp (t)-1}{t}\left(\frac{t}{K} \frac{\exp \left(\frac{t}{K}\right)}{\exp \left(\frac{t}{K}\right)-1}-1\right) \mathrm{d} t
$$

for $K>0$. If $0 \leq z \leq \pi K$, then

$$
\left|T_{K}(z)+\frac{z}{2 K}\right| \leq \frac{4 \exp (z)}{K} .
$$

Proof: This is basically Lemma 11 in Manstavičius and Petuchovas (2016).

Theorem 2.2 can now be proved.

Proof of Theorem 2.2: We only need to verify the asymptotics of the terms $h_{n, \alpha}^{\prime}(1)$ and $h_{n, \alpha}^{\prime}(1)+h_{n, \alpha}^{\prime \prime}(1)$. Concerning $h_{n, \alpha}^{\prime}(1)$, by Proposition 4.8 and a substitution, the important term is

$$
\begin{aligned}
& \int_{0}^{\alpha(n) \log \left(x_{n, \alpha}(1)\right)} \frac{\exp (v)-1}{v} \frac{v}{\alpha(n)} \frac{\exp \left(\frac{v}{\alpha(n)}\right) \mathrm{d} v}{\exp \left(\frac{v}{\alpha(n)}\right)-1} \\
= & T_{\alpha(n)}\left(\alpha(n) \log \left(x_{n, \alpha}(1)\right)\right)+I\left(\alpha(n) \log \left(x_{n, \alpha}(1)\right)\right)
\end{aligned}
$$

where

$$
I(z):=\int_{0}^{z} \frac{\exp (t)-1}{t} \mathrm{~d} t .
$$

Since $0 \leq \alpha(n) \log \left(x_{n, \alpha}(1)\right) \leq \pi \alpha(n)$ for $n$ large enough $\left(\log \left(x_{n, \alpha}(1)\right) \rightarrow 0\right.$ for $n \rightarrow \infty$ by Lemma 4.11 and the assumptions about the growth of $\alpha(n)$ in (2.7)), we can apply Lemma 4.12. The resulting terms

$$
\frac{\alpha(n) \log \left(x_{n, \alpha}(1)\right)}{2 \alpha(n)}=\frac{\log \left(x_{n, \alpha}(1)\right)}{2} \longrightarrow 0
$$

and

$$
\begin{aligned}
\frac{4 \exp \left(\alpha(n) \log \left(x_{n, \alpha}(1)\right)\right)}{\alpha(n)} & =O\left(\frac{\exp \left(\xi\left(\frac{n}{\alpha(n)}\right)\right)}{\alpha(n)}\right) \\
& =O\left(\frac{n}{(\alpha(n))^{2}} \log (\alpha(n)+2)\right)
\end{aligned}
$$

(by Lemma 4.11) do not contribute to the asymptotic expansion by the assumptions concerning $\alpha(n)$ in (2.7).

The integrand

$$
\frac{\exp (v)-1}{v}
$$

in $I$ is strictly increasing in $v$. This fact,

$$
\alpha(n) \log \left(x_{n, \alpha}(1)\right)=\xi\left(\frac{n}{\alpha(n)}\right)+O\left(\frac{\log \left(\frac{n}{\alpha(n)}+1\right)}{\alpha(n)}\right)
$$


(by Lemma 4.11), and

$$
I^{\prime}(\xi(u))=u
$$

(by Equation (2.6)) lead to

$$
\begin{aligned}
I\left(\alpha(n) \log \left(x_{n, \alpha}(1)\right)\right) & =I\left(\xi\left(\frac{n}{\alpha(n)}\right)\right)+O\left(\frac{n}{\alpha(n)} \frac{\log \left(\frac{n}{\alpha(n)}+1\right)}{\alpha(n)}\right) \\
& =I\left(\xi\left(\frac{n}{\alpha(n)}\right)\right)+O\left(\frac{n}{(\alpha(n))^{2}} \log \left(\frac{n}{\alpha(n)}+1\right)\right) .
\end{aligned}
$$

where the error term is again of lower order. A calculation similar to Equation (4.18) which additionally applies Equation (2.6) leads to

$$
I\left(\xi\left(\frac{n}{\alpha(n)}\right)\right) \sim \frac{n}{\alpha(n)} \sum_{k=0}^{\infty} \frac{k !}{\left(\xi\left(\frac{n}{\alpha(n)}\right)\right)^{k}} .
$$

Since the corresponding error terms are of lower order,

$$
h_{n, \alpha}^{\prime}(1) \sim \frac{n}{\alpha(n)} \sum_{k=0}^{\infty} \frac{k !}{\left(\xi\left(\frac{n}{\alpha(n)}\right)\right)^{k}}
$$

follows.

As to $h_{n, \alpha}^{\prime \prime}(1)$, we have

$$
\begin{aligned}
\alpha(n)\left(x_{n, \alpha}(1)-1\right) & =\alpha(n) \log \left(x_{n, \alpha}(1)\right)+O\left(\alpha(n)\left(x_{n, \alpha}(1)-1\right)^{2}\right) \\
& =\xi\left(\frac{n}{\alpha(n)}\right)+O\left(\frac{\left(\xi\left(\frac{n}{\alpha(n)}\right)\right)^{2}}{\alpha(n)}+\frac{\log \left(\frac{n}{\alpha(n)}+1\right)}{\alpha(n)}\right)
\end{aligned}
$$

by Taylor's theorem and Lemma 4.11. The order of the error term is such that applying Equation (4.25) to the result in Proposition 4.9 yields

$$
h_{n, \alpha}^{\prime \prime}(1) \sim-\frac{n}{\alpha(n)} \sum_{k=0}^{\infty} \frac{1}{\left(\xi\left(\frac{n}{\alpha(n)}\right)\right)^{k}} .
$$

The claim follows.

\section{Acknowledgements}

The authors wish to thank Dirk Zeindler for helpful discussions. The authors also wish to thank the anonymous referees for their very helpful and detailed comments.

\section{References}

R. Arratia and S. Tavaré. The cycle structure of random permutations. Ann. Probab. 20 (3), 1567-1591 (1992). MR1175278.

V. Betz. Random permutations of a regular lattice. J. Stat. Phys. 155 (6), 12221248 (2014). MR3207736.

V. Betz and D. Ueltschi. Spatial random permutations and Poisson-Dirichlet law of cycle lengths. Electron. J. Probab. 16, no. 41, 1173-1192 (2011). MR2820074. 
V. Betz, D. Ueltschi and Y. Velenik. Random permutations with cycle weights. Ann. Appl. Probab. 21 (1), 312-331 (2011). MR2759204.

L. V. Bogachev and D. Zeindler. Asymptotic statistics of cycles in surrogate-spatial permutations. Comm. Math. Phys. 334 (1), 39-116 (2015). MR3304271.

J. M. DeLaurentis and B. G. Pittel. Random permutations and Brownian motion. Pacific J. Math. 119 (2), 287-301 (1985). MR803120.

DLMF. NIST Digital Library of Mathematical Functions. 6.2.E5, 6.12.E2, Release 1.0.10 of 2015-08-07. Online companion to Olver et al. (2010).

P. Flajolet and R. Sedgewick. Analytic combinatorics. Cambridge University Press, Cambridge (2009). ISBN 978-0-521-89806-5. MR2483235.

V. Gončarov. On the field of combinatory analysis. Amer. Math. Soc. Transl. (2) 19, 1-46 (1962). MR0131369 Translation of the paper originally published in Soviet Math. Izv., Ser. Math.8, 3-48 (1944).

S. Grosskinsky, A. A. Lovisolo and D. Ueltschi. Lattice permutations and PoissonDirichlet distribution of cycle lengths. J. Stat. Phys. 146 (6), 1105-1121 (2012). MR2903040.

J. C. Hansen. A functional central limit theorem for the Ewens sampling formula. J. Appl. Probab. 27 (1), 28-43 (1990). MR1039182.

V. F. Kolčin. A certain problem of the disbribution of particles in cells, and cycles of random permutation. Teor. Verojatnost. i Primenen. 16, 67-82 (1971). MR0283840.

E. Manstavičius and R. Petuchovas. Permutations without long or short cycles. Electronic Notes in Discrete Mathematics 49, 153 - 158 (2015). DOI: 10.1016/j.endm.2015.06.023.

E. Manstavičius and R. Petuchovas. Local probabilities for random permutations without long cycles. Electron. J. Combin. 23 (1), Paper 1.58, 25 (2016). MR3484763.

K. Maples, A. Nikeghbali and D. Zeindler. On the number of cycles in a random permutation. Electron. Commun. Probab. 17, no. 20, 13 (2012). MR2943103.

J. D. Murray. Asymptotic analysis, volume 48 of Applied Mathematical Sciences. Springer-Verlag, New York, second edition (1984). ISBN 0-387-90937-0. MR740864.

A. Nikeghbali, J. Storm and D. Zeindler. Large cycles and a functional central limit theorem for generalized weighted random permutations. ArXiv Mathematics eprints (2013). arXiv: 1302.5938.

F. W. J. Olver, D. W. Lozier, R. F. Boisvert and C. W. Clark, editors. NIST Handbook of Mathematical Functions. U.S. Department of Commerce, National Institute of Standards and Technology, Washington, DC; Cambridge University Press, Cambridge (2010). Print companion to DLMF. MR2723248.

V. N. Sachkov. Probabilistic methods in combinatorial analysis, volume 56 of Encyclopedia of Mathematics and its Applications. Cambridge University Press, Cambridge (1997). ISBN 0-521-45512-X. MR1453118.

L. A. Shepp and S. P. Lloyd. Ordered cycle lengths in a random permutation. Trans. Amer. Math. Soc. 121, 340-357 (1966). MR0195117.

A. L. Yakymiv. A limit theorem for the total number of cycles of a random $A$ permutation. Teor. Veroyatn. Primen. 52 (1), 69-83 (2007a). MR2354570.

A. L. Yakymiv. Random $A$-permutations: convergence to a Poisson process. Mat. Zametki 81 (6), 939-947 (2007b). MR2349109. 